\title{
A Combined Algorithm Approach for PD Location Estimation Using RF Antennas
}

\author{
José Manuel Fresno, Guillermo Robles, Juan Manuel \\ Martínez-Tarifa \\ Department of Electrical Engineering \\ Universidad Carlos III de Madrid \\ Madrid, Spain \\ jfresno@ing.uc3m.es, grobles@ing.uc3m.es, \\ jmmtarif@ing.uc3m.es
}

\author{
Brian G Stewart \\ Institute of Energy and Environment \\ Department of Electronic and Electrical Engineering \\ University of Strathclyde \\ Glasgow, Scotland \\ brian.stewart.100@strath.ac.uk
}

\begin{abstract}
To locate the positions of partial discharge sources in free space at least four RF antennas are arranged in a suitable spatial geometry to detect the radiated electromagnet energy from the discharge. The time-difference-of-arrival (TDOA) between the signals from each antenna are then used within multi-lateration equations to determine the position of the source. The iterative Hyperbolic Least Squares (HLS) method and the non-iterative Maximum Likelihood Estimator (MLE) method are two common techniques used in the literature to solve the multi-lateration equations. This paper investigates the ability of combining MLE and HLS to improve location accuracy and maintain fast location computation time. To this end HLS, MLE and the combined MLEHLS method are evaluated in terms of location accuracy and computation performance for three spatial antenna configurations, namely Square, Pyramidal and Trapezoidal arrangements. The location accuracies for each method are evaluated for theoretical TDOA values and also for the case when a finite sampling rate of $10 \mathrm{G}$ samples-per-second is considered; the latter is implemented through appropriate rounding up of TDOA values by one sample time. It is shown that MLE-HLS produces improved location accuracy compared with HLS and MLE for both theoretical and finite sampled TDOA values. In addition, it is shown that MLE-HLS improves significantly the computation time over the iterative HLS method.
\end{abstract}

Keywords - radio-frequency localization; partial discharges; location algorithms

\section{INTRODUCTION}

High voltage equipment undergoes a variety of stresses including thermal, mechanical and electrical stresses, which can all play a part in accelerating the aging and degradation of electrical insulation thus leading to equipment failure. Consequently, condition assessment of electrical insulation has increasingly become a critical part of asset maintenance strategies [1, 2]. In this regard, partial discharge (PD) measurements have become an important part of predicative monitoring for high voltage equipment [3]. A PD is an impulse current pulse within electrical insulation that occurs as the insulation starts to break down. Due to the acceleration of charge within impulse currents, PD pulses are a source of broadband radiated electromagnetic energy, which may cause electromagnetic interference (EMI) from the low $\mathrm{MHz}$ region up to and beyond $1 \mathrm{GHz}$. A number of PD surveillance methods use radio antennas to detect the presence of these signals and to evaluate the severity of the electrical insulation [4].

Often the location of the PD source may be successfully determined from the detected radiated energy within substation environments $[5,6]$. It is possible to locate the position of a PD source in free space from the RF emissions using an array of antennas with knowledge of each antenna's spatial coordinates. The time-differences-of-arrival (TDOA) of RF emissions arriving at the spatially positioned antennas then permits the application of multi-lateration methods to estimate the PD source location.

From the published literature, a number of algorithms may be used with TDOA measurements to estimate the location of PD sources. Two of these algorithms are the Hyperbolic Least Squares (HLS) algorithm and the Maximum Likelihood Estimator (MLE) algorithm [7, 8, 9]. HLS is an iterative algorithm but has a high dependency on the initialization parameters and therefore in some cases is unable to provide an iterative accurate solution. In addition, its execution time, based on the number of iterations required to achieve certain accuracy, may be lengthy depending on the initial iterative parameters. MLE is a non-iterative Global Positioning System (GPS) location algorithm that provides two possible solutions based on directly solving the TDOA multi-lateration equations. One of the MLE solutions is the best solution and the other is a solution in the coordinate domain space with the same TDOA solution but is incorrect.

In this paper, a combined method of employing MLE and HLS is proposed which enables the correct MLE solution to be determined based on the HLS objective function. Through simulations, the behavior of the combined MLE-HLS method is compared with MLE and HLS independently in relation to accuracy of source location and location computation time. As demonstrated in previous published work, the spatial antenna arrangement itself, and also finite signal sampling issues, may adversely affect source location accuracy for different location algorithms [10]. Therefore, the combined algorithm, and the individual algorithms are evaluated and compared for three commonly used antenna configurations, namely Square, Pyramidal and Trapezoidal arrangements in order to demonstrate the improved ability of the combined algorithm 
approach. In addition, simulations are carried out for both theoretical TDOA values and TDOA values that have been rounded up based on a finite sampling rate of $10 \mathrm{G}$ samples $\mathrm{s}^{-1}$.

The structure of the paper is as follows. Section 2 describes the principles of multi-lateration location and summarizes briefly the HLS and MLE algorithms and the principles of the combined MLE-HLS approach. Section 3 presents examples of theoretical TDOA simulations and 10G samples s ${ }^{-1}$ TDOAs of PD source location estimation and computational times for Square, Pyramidal and Trapezoidal antenna arrangements within a selected size volume of source positions. Section 4 is a conclusions section and summarizes the main points from the work presented in the paper.

\section{MULTI-LATERATION AND LOCATION ESTIMATION ALGORITHMS}

Assume within a Cartesian coordinate system that an unknown PD emission occurs at position $\boldsymbol{P}_{s}=\left(x_{s}, y_{s}, z_{s}\right)$, where $x_{s}, y_{s}$, and $z_{s}$ are the Cartesian coordinate PD source positions. Also, assume that four antennas are located at known positions $\boldsymbol{P}_{i}=\left(x_{i}, y_{i}, z_{i}\right), i=\{1,2,3,4\}$ where $x_{i}, y_{i}$, and $z_{i}$ are the coordinates of the $i^{\text {th }}$ antenna. Then the distance travelled by the PD signal to the $i^{\text {th }}$ antenna is given by Equation (1):

$$
D_{i}=\sqrt{\left(x_{i}-x_{s}\right)^{2}+\left(y_{i}-y_{s}\right)^{2}+\left(z_{i}-z_{s}\right)^{2}}=c t_{i}
$$

where $c$ is the speed of light, and $t_{i}$ is the time of propagation from the PD source to antenna $i^{\text {th }}$. Multi-lateration is the process of solving the non-linear Equation (1) to estimate the PD source location using the time of arrival of signals at each antenna and the known antenna positions. The HLS, MLE and MLE-HLS methods for PD source location estimation are described below.

\section{A. Hyberbolic Least Squares (HLS)}

In the HLS algorithm the main assumption is that the distance between the $j^{\text {th }}$ antenna and the PD source minus the distance between the $i^{\text {th }}$ antenna and the PD source is equal to the TDOA between the $j^{\text {th }}$ and $i^{\text {th }}$ antennas multiplied by the speed propagation [6]:

$$
D_{j}-D_{i}=c t_{j i}
$$

where $t_{j i}=\left(t_{j}-t_{i}\right)$. Considering the four-antenna spatial arrangement, for TDOAs referenced for example to antenna 1, the coordinates $\left(x_{s}, y_{s}, z_{s}\right)$ are estimated using least squares, minimizing the following objective function $S$ through iterative techniques:

$$
S\left(\widehat{x_{s}}, \widehat{y_{s}}, \widehat{z_{s}}\right)=\sum_{i=2}^{4}\left(\widehat{D}_{i}-\widehat{D}_{1}-c t_{i 1}\right)^{2} .
$$

To solve (3) when the PD source is unknown, then $(0,0,0)$ is often a suitable starting position for the iterative solution equations.

\section{B. Maximum Liklihood Estimator (MLE)}

The MLE solution is an optimized solution that requires solving directly the quadratic Equation (4) [7].

$$
\begin{aligned}
& {\left[\begin{array}{l}
\hat{x}_{S} \\
\hat{y}_{S} \\
\hat{z}_{S}
\end{array}\right]=-\left[\begin{array}{lll}
x_{2}-x_{1} & y_{2}-y_{1} & z_{2}-z_{1} \\
x_{3}-x_{1} & y_{3}-y_{1} & z_{3}-z_{1} \\
x_{4}-x_{1} & y_{4}-y_{1} & z_{4}-z_{1}
\end{array}\right]^{-1} } \\
& \times\left\{\left[\begin{array}{l}
D_{21} \\
D_{31} \\
D_{41}
\end{array}\right] \widehat{D}_{1}+\frac{1}{2}\left[\begin{array}{l}
D_{21}^{2}-K_{2}+K_{1} \\
D_{31}^{2}-K_{3}+K_{1} \\
D_{41}^{2}-K_{4}+K_{1}
\end{array}\right]\right\}
\end{aligned}
$$

where $K_{i}=x_{i}^{2}+y_{i}^{2}+z_{i}^{2}$ with $i=\{1,2,3,4\}$ and $\widehat{D}_{1}$ is defined by:

$$
\widehat{D}_{1}=\sqrt{\hat{x}_{s}^{2}+{\hat{y}_{s}}^{2}+\hat{z}_{s}^{2}-2 \hat{x}_{s} x_{1}-2 \hat{y}_{s} y_{1}-2 \hat{z}_{s} z_{1}+K_{1}} \text {. }
$$

Two direct solutions are possible, which will be referred to as the positive root, $\mathrm{MLE}^{+}$, and the negative root, $\mathrm{MLE}^{-}$, one of which will be erroneous. The issue of resolving the correct solution leads to the following combined method.

\section{MLE-HLS}

For this method, the preferred MLE solution is determined by using the objective function of Equation (6) based on HLS. In MLE-HLS, the MLE solution that provides the minimal objective function value is chosen as the correct solution.

$$
S\left(\hat{x}_{s}, \hat{y}_{s}, \hat{z}_{s}\right)=\sum_{i=1}^{3} \sum_{j=i+1}^{4}\left(\widehat{D}_{i}-\widehat{D}_{j}-\tau_{i j} c\right)^{2} .
$$

\section{SIMULATION RESULTS}

Details of the chosen four antenna positions for the Square,

\begin{tabular}{|c|c|c|c|c|c|}
\hline \multirow{2}{*}{\multicolumn{2}{|c|}{ Arrangement }} & \multicolumn{4}{|c|}{ Antenna Positions (m) } \\
\hline & & \multirow{2}{*}{$\begin{array}{c}\text { Antenna } 1 \\
-1.0\end{array}$} & \multirow{2}{*}{$\begin{array}{c}\text { Antenna } 2 \\
-1.0\end{array}$} & \multirow{2}{*}{$\begin{array}{c}\text { Antenna } 3 \\
+1.0\end{array}$} & \multirow{2}{*}{$\begin{array}{c}\text { Antenna } 4 \\
+1.0\end{array}$} \\
\hline \multirow{3}{*}{ Square } & $x$ & & & & \\
\hline & $y$ & -1.0 & +1.0 & -1.0 & +1.0 \\
\hline & $z$ & 0 & 0 & +2.0 & 0 \\
\hline \multirow{3}{*}{$\begin{array}{c}\text { Pyrami } \\
\text {-dal }\end{array}$} & $x$ & $-\sqrt{3} / 3$ & $-\sqrt{3} / 3$ & $+2 \sqrt{3} / 3$ & 0 \\
\hline & $y$ & -1.0 & +1.0 & 0 & 0 \\
\hline & $z$ & 0 & 0 & 0 & $-2 \sqrt{2 / 3}$ \\
\hline \multirow{3}{*}{$\begin{array}{c}\text { Trapez } \\
\text {-oidal }\end{array}$} & $x$ & $-2 / 3$ & $+2 / 3$ & $+2 / 3$ & $-2 / 3$ \\
\hline & $y$ & -2.0 & -1.0 & +1.0 & +2.0 \\
\hline & $z$ & 0 & +2.0 & 0 & 0 \\
\hline
\end{tabular}
Pyramidal and Trapezoidal arrangements are shown in Table I. It should be noted that for 3D spatial resolution solutions to be achieved, one of the four antennas requires to be positioned out of the plane formed by the three other antennas. Each antenna array is centered on the origin of the coordinate system.

TABLE I. ANTENNA CONFIGURATIONS AND ANTENNA POSITIONS

Theoretical PD source positions were simulated within the volume of a cube with dimensions of $-10 m \leq(x, y) \leq+10 \mathrm{~m}, 0 \mathrm{~m}$ $\leq z \leq+10 \mathrm{~m}$ and positioned at locations separated by $2 \mathrm{~m}$. In total 726 theoretical PD source positions are considered. The estimated PD location results for HLS, MLE and MLE-HLS are determined using both the theoretical TDOA values from each PD source position (effectively infinite sampling), and also for TDOA values which are rounded up appropriately to the nearest 
sampling point presuming a sampling rate of $10 \mathrm{G}$ samples $\mathrm{s}^{-1}$. The error on the location estimation regarding the actual position is determined through Equation (7):

$$
\epsilon=\sqrt{\left(\widehat{x_{s}}-x_{s}\right)^{2}+\left(\widehat{y_{s}}-y\right)^{2}+\left(\widehat{z_{s}}-z_{s}\right)^{2}} .
$$

All location algorithms were implemented in the Matlab environment and executed using an Intel i7 processor running Matlab under real-time priority conditions ensuring that the processor CPU time was dedicated to the location estimation algorithms. Clearly different processors will produce different computational times, however, the evaluated times for each algorithm using this approach produces a comparative evaluation of the expected speeds of computation.

As examples of location accuracy, Fig. 1 and Fig. 2 give a visual presentation of the location results over all considered PD source locations within the chosen spatial volume for theoretical TDOA evaluations using the HLS and MLE-HLS algorithms respectively for the Square antenna arrangement. The error, $\varepsilon$, on each location is also shown using a color-grading scheme i.e. green $\leq 0.01 \mathrm{~m}, 0.01 \mathrm{~m}<$ yellow $\leq 0.2 \mathrm{~m}, 0.2 \mathrm{~m}<$ orange $\leq 1 \mathrm{~m}$ and red $>1 \mathrm{~m}$.

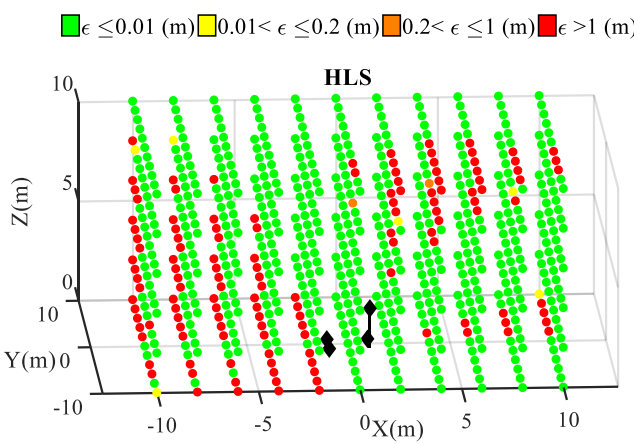

Fig. 1 Simulated PD source points and associated location errors for theoretical TDOA evaluations using HLS for the Square antenna configuration.

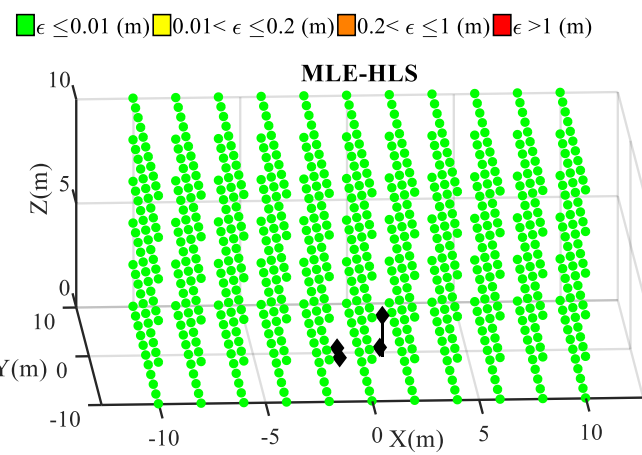

Fig. 2 Simulated PD source points and associated location errors for theoretical TDOA evaluations using MLE-HLS for the Square antenna configuration.

It can be seen in Fig. 1. that all PD source positions have been iterated to a solution, but a range of accuracies exist within the volume. In contrast, Fig. 2 shows how MLE-HLS provides high accuracy for all PD positions analyzed. Figs. 3, 4 and 5 display (a) the computational times for location estimation, (b) the location with errors for theoretical TDOAs, and (c) the location with errors for $10 \mathrm{G}$ sampled s ${ }^{-1}$ TDOAs for the Square, Pyramidal and Trapezoidal antenna arrangements respectively.

\section{Square Configuration}

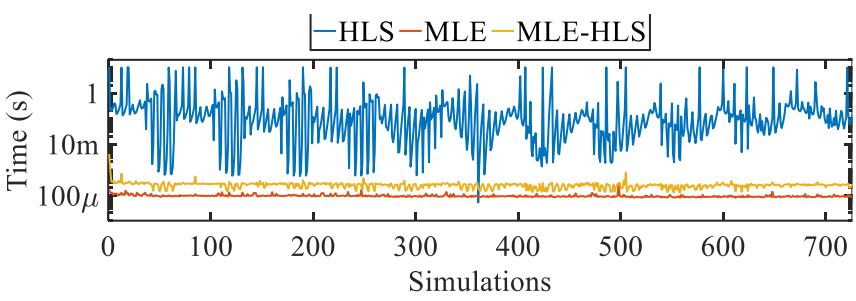

(a) Computational time to produce location estimates

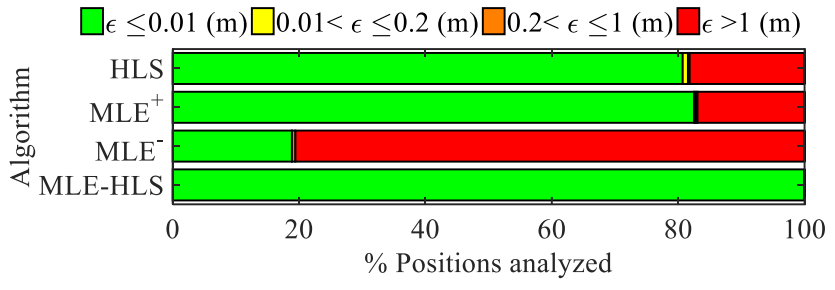

(b) Location accuracies for theoretical TDOA values

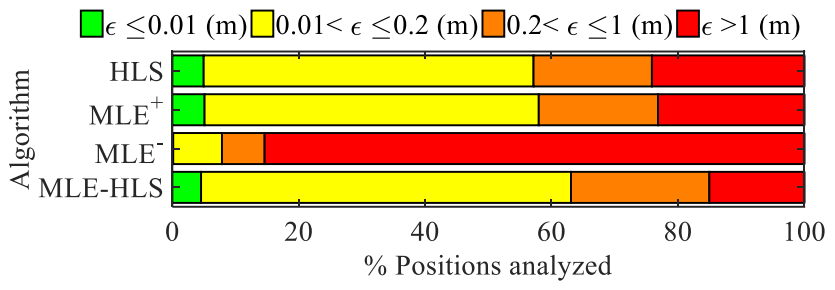

(c) Location accuracies for rounded up sampled TDOA values

Fig. 3 Square configuration results for HLS, MLE ${ }^{+}$, MLE ${ }^{-}$and MLE-HLS

\section{Pyramidal Configuration}

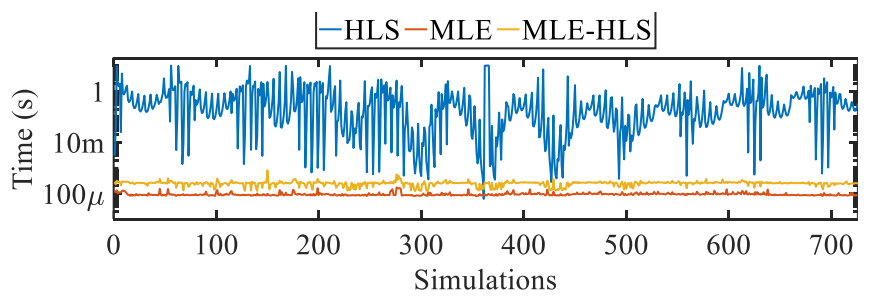

(a) Computational time to produce location estimates

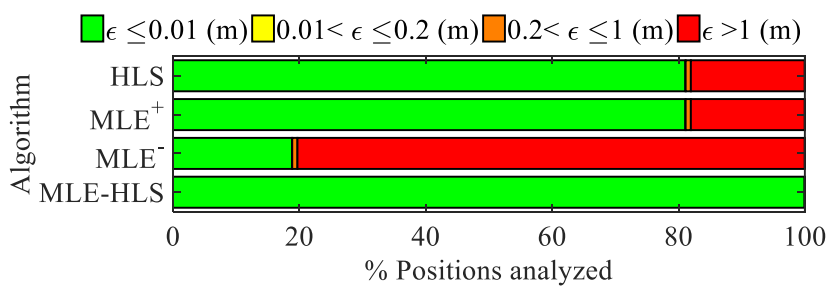

(b) Location accuracies for theoretical TDOA values

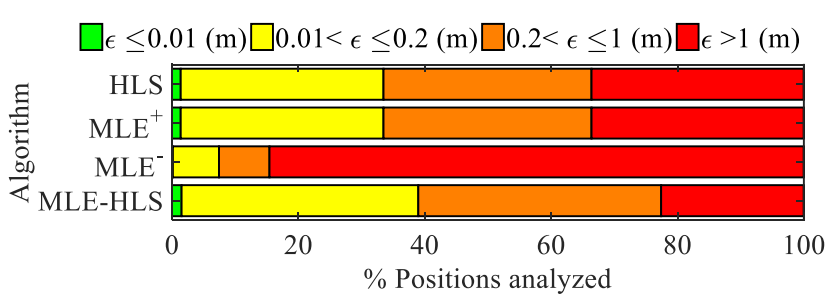

(c) Location accuracies for rounded up sampled TDOA values

Fig. 4 Pyramidal configuration results for HLS, $\mathrm{MLE}^{+}$, MLE${ }^{-}$and MLE-HLS 


\section{Trapezoidal Configuration}

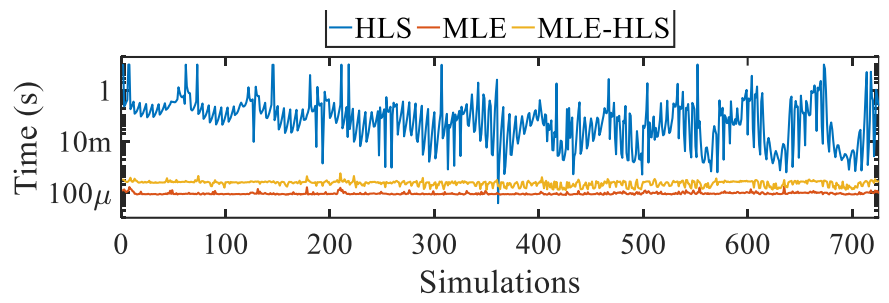

(a) Computational time to produce location estimates

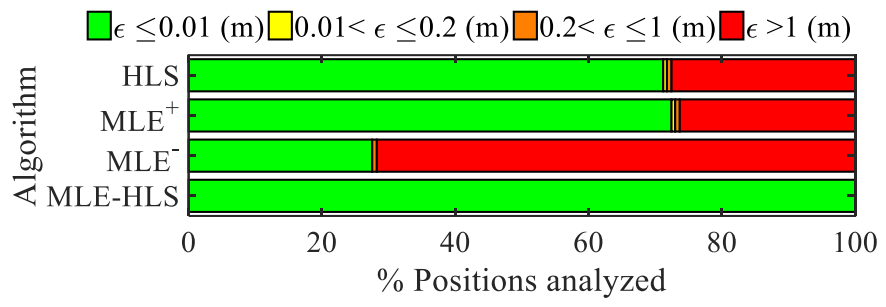

(b) Location accuracies for theoretical TDOA values

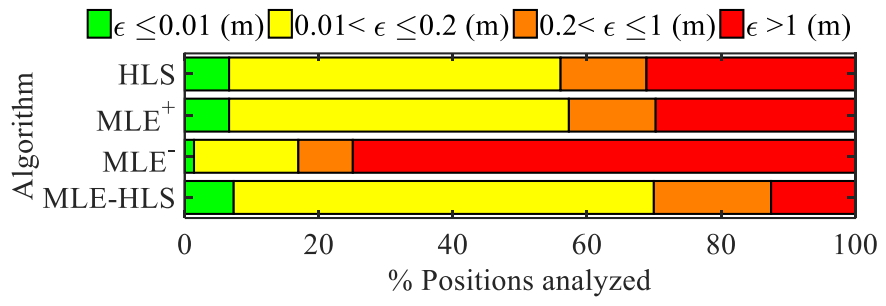

(c) Location accuracies for rounded up sampled TDOA values

Fig. 5 Trapezoidal configuration results for HLS, $\mathrm{MLE}^{+}, \mathrm{MLE}^{-}$and MLE-HLS

The results show that for theoretical TDOA evaluations the MLE-HLS method produces $100 \%$ location accuracies of less than $0.01 \mathrm{~m}$ for all three antenna configurations. MLE-HLS also ensures in each situation that the correct MLE solution is always selected thus removing the ambiguity between $\mathrm{MLE}^{+}$and $\mathrm{MLE}^{-}$ solutions. There is a very small increase in computation time $\left(<10^{-4} s\right)$ required for MLE-HLS when compared to MLE on its own, but this may be presumed to be insignificant in the context of location estimation. The small computational times for MLEHLS also compares much more favourably to the comparatively lengthy computational times required for HLS location estimation. In addition, HLS cannot guarantee an accurate location estimation as the method depends on the initial set up HLS iterative conditions.

When errors due to rounding up of theoretical TDOA values as a consequence of finite sampling are considered, it can be seen that MLE-HLS still produces the highest accuracy of location estimation when compared with the other methods, with around $80 \%$ and above estimations for all antenna configurations being less than $1 \mathrm{~m}$ in accuracy.

In terms of computation time for HLS, as the simulations are processed for each position in a systematic and logical way, it can be seen that patterns of variable computational behaviour are evident. Clearly some iterative solutions are established quickly, whilst others take much longer. This behaviour is removed when using the MLE-HLS approach.

\section{CONCLUSIONS}

This paper has shown that a combined MLE-HLS PD location estimation approach will improve the location accuracy of determined PD sources for RF antenna multi-lateration when compared to HLS and MLE methods. This has been established for three commonly used antenna arrangements comprising four antennas and for both theoretical and finite sampled TDOA estimations. In terms of location accuracy using MLE-HLS, the results show that the Square and Trapezoidal configurations are to be preferred over the Pyramidal configuration.

A further key result from the simulations is that not only is a high sampling rate to be preferred for accurate location estimation, but also that the use of MLE-HLS enables a fast, robust and improved accuracy method for PD location estimation even when finite sampling of signals is utilised.

\section{ACKNOWLEDGMENT}

The work undertaken in this paper has been funded by the Spanish Government under contract DPI2015-66478-C2-1. Jose M. Fresno received an international travel grant from the Universidad Carlos III of Madrid.

\section{REFERENCES}

[1] W.H. Bartley, "Life cycle management of utility transformer assets", Breakthrough Asset Management for the Restructured Power Industry Conf., Utah, October 2002.

[2] X. Zhang, E. Gockenbach, V. Wasserberg, and H. Borsi, "Estimation of the Lifetime of the Electrical Components in Distribution Networks", IEEE Transactions on Power Delivery, pp. 515-522, 2007.

[3] IEEE Guide for the Measurement of Partial Discharges in AC Electric Machinery. IEEE Std 1434-2014, pp. 1-89, 2014.

[4] A. Nesbitt, B.G. Stewart, S.G. McMeekin, S. Conner, J.C. Gamio, K. Liebech-Lien, H.O. Kristiansen, and S. Kråkenes "A Novel Approach to High Voltage Substation Surveillance using Radio Frequency Interference Measurement", IEEE Electrical Insulation Conference, pp. 159-163, 2009.

[5] P.J. Moore, I.E. Portugues, and I. Glover, "Radiometric location of partial discharge sources on energized high-voltage plant", IEEE Transactions on Power Delivery, vol.20, pp. 2264-2272, 2005.

[6] B.G. Stewart, A. Nesbitt, A., and L. Hall, "Triangulation and 3D location estimation of RFI and Partial Discharge sources within a $400 \mathrm{kV}$ substation", IEEE Electrical Insulation Conference, pp. 164-168, 2009.

[7] L. Tang, Z. Wu, H. Li, and D. Nie, "Location of partial discharges in power transformers using computer-aided acoustic techniques", Canadian Journal of Electrical and Computer Engineering, vol. 21, pp. 67-71, 1996.

[8] Y.T. Chan, K.C. Ho, "A simple and efficient estimator for hyperbolic location", IEEE Transactions on Signal Processing, vol. 42(8), pp. 19051915, 1994.

[9] O. El Mountassir, B.G. Stewart, A.J. Reid, S.G. McMeekin, "Quantification of the performance of iterative and non-iterative computational methods of locating partial discharges using RF measurement techniques", Electric Power Systems Research, vol. 143, pp. 110-120, 2017.

[10] G. Robles, G.; J.M. Fresno, M. Sánchez-Fernández, and J.M. MartínezTarifa, "Antenna deployment for the localization of partial discharges in open-air substations", Sensors, vol.16, 541, 2016 